【研究简报】

\title{
双镥金属富勒烯的合成、提取与表征
}

\author{
郝春雁 郭兴华 刘子阳 刘淑莹* \\ (中国科学院长春应用化学研究所, 长春 $130022 . *$ 联系人)
}

\section{关链词双镂金属言勒㜓 $\mathrm{Lu}_{2} \mathrm{C}_{2 n}$ 高压提取 LDi-MS DEI-MS}

自 $\mathrm{La} @ \mathrm{C}_{82}$ 被发现以来, 能导致新分子、新材料不断涌现的金属富勒㛓的研究就一直是富 勒烯化学领域中最吸引人的分支 ${ }^{[1,2]}$, 但是它的发展比较缓慢, 直到 $\mathrm{K}-\mathrm{H}$ 方法被应用于宏观量 地合成金属富勒㛓, 这种情况才有所改善 ${ }^{[3]}$. 现在, 大多数铜系元素, 包括 $\mathrm{La}, \mathrm{Ce}, \mathrm{Nd}, \mathrm{Sm}$, $\mathrm{Eu}, \mathrm{Gd}, \mathrm{Tb}, \mathrm{Dy}, \mathrm{Ho}$ 和 $\mathrm{Er}$, 已通过金属/碳棒在低压氨气条件下的电弧放电过程被成功地包 襄于富勒㛓碳笼当中并被溶剂提取出来 ${ }^{[4]}$, 它们提取液的质谱图均给出明显的 $\mathrm{M}_{0} \mathrm{C}_{82}$ 信号. 与此相反, 我们这里报道了一种特殊的铜系元素——镥, 在它的金属富勒烯提取液中, 只有双 镜富勒烯, $\mathrm{Lu}_{2} @ \mathrm{C}_{2 n}$, 能被激光解吸电离飞行时间质谱与解吸电子轰击质谱观察到.

含富勒烯与双镜富勒㛓的碳煑用电弧放电法制备. 简单来说, 直径 $6 \mathrm{~mm}$ 的光谱纯碳棒, 钻孔, 填入 $99.99 \% \mathrm{Lu}_{2} \mathrm{O}_{3}$ 与碳粉的混合物, 使总原子比为 $1.0 \mathrm{Lu} / 100 \mathrm{C}$, 此金属/碳的混合棒 先在真空条件下 $\left(10^{-3} \times 133.332 \mathrm{~Pa}\right), 2000 \mathrm{~K}$ 处理 $3 \mathrm{~h}$, 然后作为正极在 $160 \times 133.332 \mathrm{~Pa}$ 氮 气中直流电弧放电, 电弧电流 $80 \mathrm{~A}$. 生成的碳炱用甲苯索氏提取后, 再于高压金中 $523 \mathrm{~K}$ 时,

$\left.v^{\prime}=0\right)$ 的猝灭速率常数也按 $\mathrm{Cl}, \mathrm{Br}, \mathrm{I}$ 的顺序依次增加. 说明试剂分子中的卤素对 $\mathrm{PCl}\left(\mathrm{b}, v^{\prime}=\right.$ $0)$ 的猝灭也有重要的影响.

以上这些规律和前人研究的 $\mathrm{NF}(\mathrm{b}), \mathrm{PF}(\mathrm{b})$ 和 $\mathrm{NCl}(\mathrm{b})^{[3,5]}$ 的猝灭规律基本一致. 对这些 规律更深入的讨论将另文发表.

致谢本工作为国家自然科学基金(批准号:29473105)资助项目.

\section{参 考 献}

1 Coxon J A, Wickramaaratchi M A. Visible and near infared emission spectra of $\mathrm{PCl}$ excited in the reaction of $\operatorname{Ar}\left({ }^{3} \mathrm{P}_{0.2}\right)$ with $\mathrm{PCl}_{3}$. Journal of Molecular Spectroscopy. 1971, 68: 372

2 Bielefeld $M$, Elfers G, Fink E H, et al. $\mathrm{O}_{2}\left(\mathrm{a}^{1} \Delta_{\S}\right)$-sensitized chemiluminescence of $\mathrm{a}^{1} \Delta \rightarrow \mathrm{X}^{3} \Sigma^{-}$and $\mathrm{b}^{1} \Sigma^{+} \rightarrow \mathrm{X}^{3} \Sigma^{-}$transitions of group V-group V and group V-group $V$ diatomic molecules. Journal of Photochemistry, 1984, 25: 419

3 Zhao Yao, Setser D W. Quenching rate constants for $\mathrm{NCl}\left(b^{1} \Sigma^{+}\right)$and $\mathrm{PCl}\left(b^{t} \Sigma^{+}\right)$and radiative lifetime of $\mathrm{NCl}\left(b^{1} \Sigma^{+}\right), \mathrm{PCl}$ $\left(b^{1} \Sigma^{+}\right)$and $\operatorname{PBr}\left(b^{1} \Sigma^{+}\right)$. J Chem Soc, Faraday Trans, 1995, 91: 2979

4 Guo Jingzhong, Gu Yueshu, Liu Chuanpu. Energy transfer process in the reaction of $\mathrm{He}\left(2^{3} \mathrm{~S}\right)$ with $\mathrm{CH}_{2} \mathrm{Cl}_{2}$. Chem Phys Lett, 1990, 169: 432

5 Zhao Yao, Setser $\mathrm{D} W$. Radiative lifetime and quenching rate constant of $\mathrm{PF}(\mathrm{b})$ and test for an electronic to vibrational energy transfer quenching mechanism. J Phys Chem, 1994, 98: 9723

(1996-08-15 收稿) 
分别以甲苯、吡啶和二硫化碳进一步高温高压提取. 各种提取液用 LDI-MS(美国 Linear-Scientific 公司 LDI-1700 质谱仪, $337 \mathrm{~nm} \mathrm{~N}$ 分子激光解吸探头上样品并使之电离, 控制激光能量较低以避 兔产生不必要碎片, 分别采集正、负离子质谱) 与 DEI-MS(英国 VG-QUATTRO 质谱仪, 电子能 量: $70 \mathrm{eV}$, 源温: $473 \mathrm{~K}$, 样品由 DCI 探头引入离子源, 解吸电流: $1400 \mathrm{~mA}$ )进行分析.

图 1 没有给出 $\mathrm{LuC}_{82}$ 的信号, 但在 $\mathrm{m} / \mathrm{z} 1334$ 处有一对应 $\mathrm{Lu}_{2} \mathrm{C}_{82}$ 的小峰; 图 2 中在 $\mathrm{m} / \mathrm{z}$ 1159 处也没有明显的 $\mathrm{LuC}_{82}$ 峰, 而 $\mathrm{m} / \mathrm{z}=1262,1286,1310 \cdots \cdots 1694$ 位置上却有一系列等 间距的谱峰. 由于只有奇数碳筷接近这些质荷比 $\left(\mathrm{C}_{105} \mathrm{~m} / \mathrm{z} 1260, \mathrm{C}_{107} \mathrm{~m} / \mathrm{z} 1284, \mathrm{C}_{109} \mathrm{~m} / \mathrm{z}\right.$ $\left.1308 \cdots \cdots \mathrm{C}_{141} \mathrm{~m} / \mathrm{z} 1692\right)$, 我们将这组峰归为 $\mathrm{Lu}_{2} \mathrm{C}_{2 n}(2 n=76 \sim 112)$. 高压提取液中, 高碳 数富勒㛓如 $\mathrm{C}_{74}(\mathrm{~m} / \mathrm{z} 888), \mathrm{C}_{84}(\mathrm{~m} / \mathrm{z} 1008), \mathrm{C}_{96}(\mathrm{~m} / \mathrm{z} 1152)$ 的相对丰度也提高了, 同位素造成 的谱峰变宽使这些高碳数富勒㛓与 $\mathrm{LuC}_{60}(\mathrm{~m} / \mathrm{z} 895), \mathrm{LuC}_{70}(\mathrm{~m} / \mathrm{z} 1015), \mathrm{LuC}_{82}(\mathrm{~m} / \mathrm{z} 1$ 159)的 分辨效果不太好, 加之有人指出激光可导致提取液中不存在物质的生成 ${ }^{[5]}$, 所以甲苯高压提 取液的 DEI 质谱 (图 3) 被用来确证上述结果. 虽然两种质谱图结果互相验证, 但它们同一谱 峰的相对强度并不相同:LDI-MS 有显著的夸张金属富勒㛓与高碳数富勒㛓的趋势, 这是由于 它们电离所需的光子数较少 ${ }^{[6]}$. 图 4 的双镜富勒烯信号非常低, 几乎消失. 图 2 与图 4 的明 显差别表明, 双镜富勒烯更易于形成正离子, 所以我们说双错富烯比空笼富勒烯具有更小的电

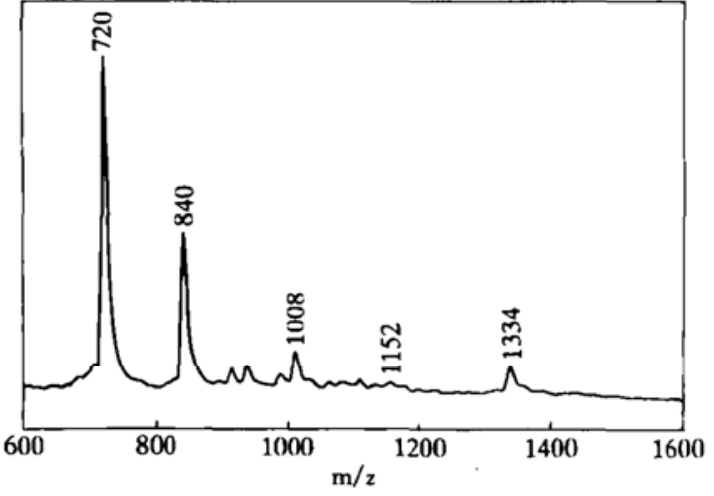

图 1 甲苯索氏提取液的正离子激光解吸电离质谱图

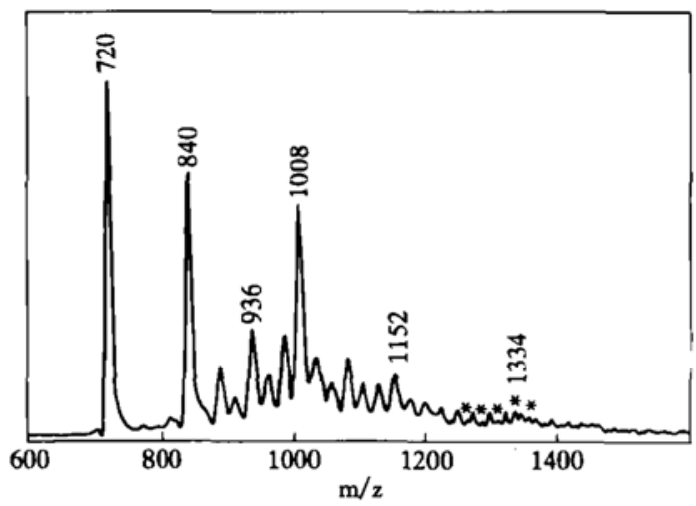

图 3 与图 2 同种提取液负离子激光解吸电离质谱图

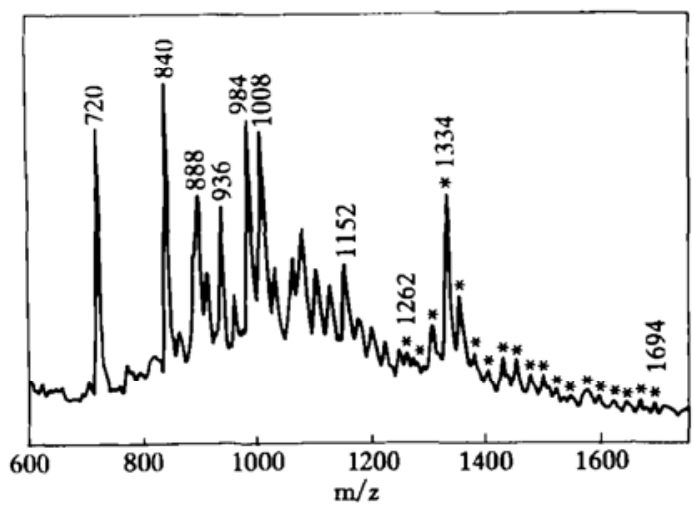

图 2 甲苯高压提取波的正离子敫光解吸电禹质诸图

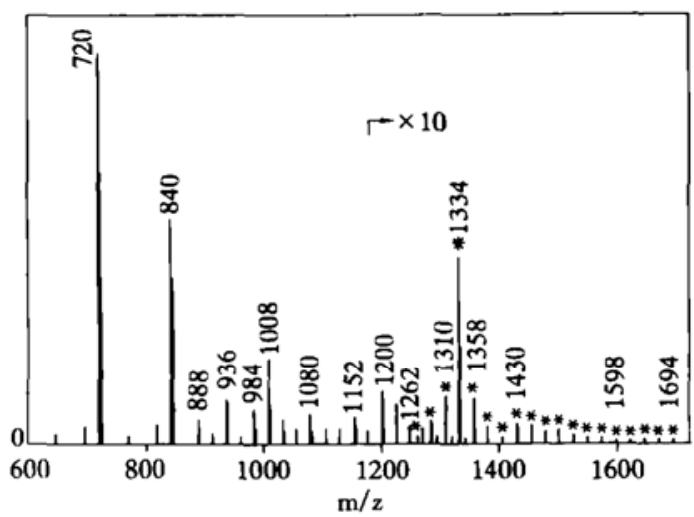

图 4 甲苯高压提取液的 DEI 质谱图 
【研究简报】

\title{
钯金属/陶瓷复合膜制备: 化学镀新过程
}

\author{
赵宏宾 李安武 谷景华 熊国兴* \\ (中国科学院大连化学物理研究所催化基础国家重点实验室, 大连 116023.*联系人)
}

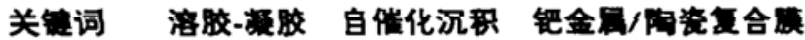

膜材料与膜过程在高技术产业中日益发挥重要的作用, 因而成为现代材料学与技术研究

离能与电子亲和势. 比较图 1 与图 2, 我们发现索氏提取液洗去了许多伴生的 $\mathrm{C}_{60}, \mathrm{C}_{70}$, 而只 提出了一部分双镜富勒烯; 第二步高温高压提取提高了双锚富勒烯的提取效率, 能提供一种高 浓度的双错富勒烯溶液, 可简化后面的分离纯化步骤。

另外, 我们还将高压提取所用的溶剂改为二硫化碳或吡啶, 改变错与碳的原子比至 $0.8 \%$ 或 $2 \%$, 但所得结果和上面相同, 并且不同于其他金属富勒烯, 双镜富勒烯在甲苯中的溶解性 要好于在二硫化碳或吡啶中 ${ }^{[7]}$. 另一方面, 我们用其他稀土重复了类似的实验, 提取液中清 楚地观察到了除 $\mathrm{LuC}_{82}$ 外的所有 $\mathrm{MC}_{82}$ 峰, 我们认为这种现象是锚本身的原因, 是由于它的电 离能及离子半径在铜系元素中最小造成的.

总之, 镂是一种特殊的铜系元素, 在电弧放电过程中它易于形成双镥富勒烯, 这种双镥富 勒烯具有比空笼富勒烯更低的电离能与电子亲和势. 并且我们所采用的两步提取法能获得相 对浓度较高的双管富勒烯提取液, 是一种新颖、高效的笼内金属富勒烯提取方法.

致谢 本工作为国家自然科学基金(批准号:59302014)资助项目.

\section{考文献}

1 Bethune D S, Johnson R D, Salem J, et al. Atoms in carbon cages: the structure and properties of endohedral fullerenes. Nature, 1993, 366(6451): 123 128

2 Heath J R, O'Brien S C, Zhang Q, et al. Lanthanum complexes of spheroidal carbon shells. J Am Chem Soc, 1985, 107 (25): $7779 \sim 7780$

3 Johnson R D, de Vries M S, Salem J, et al. Electron paramagnetic resonance studies of lanthenum-containing $\mathrm{C}_{82}$. Nature, 1992, 355(6357) : 239 240

4 Gillan E G, Yeretzian C, Min K S, et al. Endohedral rare-earth fullerenes complexes. J Phys Chem, 1992, 96(17): 6869 $\sim 6871$

5 Ross M M, Nelson, H H, Callhan J H, et al. Production and characterization of metallofullerenes. J Phys Chem, 1992, 96 (13): $5231 \sim 5234$

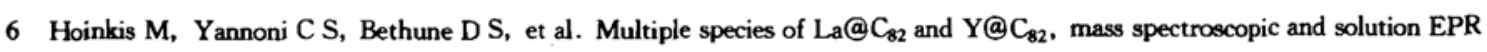
studies. Chem Phys Lett, 1992, 198(5): $461 \sim 463$

7 Shinohara H, Sato H, Ohkohchi M, et al. Encapsulation of a scandium trimer in $\mathrm{C}_{82}$. Nature, 1992, 357(6363): 52 54 